\title{
A comparison of visual and two modes of tactual letter resolution
}

\author{
JOHN R. PHILLIPS and KENNETH O. JOHNSON \\ Johns Hopkins University School of Medicine, Baltimore, Maryland \\ and \\ HELEN M. BROWNE \\ University of Melbourne, Melbourne, Australia
}

\begin{abstract}
Confusion matrices were obtained for visual letter recognition and for tactual letter recognition with (1) the finger held stationary on small raised letters and (2) the finger allowed to scan them. In each sensory mode, performance was investigated at four letter sizes, with heights ranging from 1.5 to $3.0 \mathrm{~min}$ of arc for vision and from 3.0 to $8.0 \mathrm{~mm}$ for touch. The two tactual modes yielded similar recognition performance, although performance was always better in the scanned mode. For both vision and touch, performance appeared to be limited by primary receptor spacing. Letter heights corresponding to $50 \%$ correct identification (vision, $2.2 \mathrm{~min}$ arc; scanned touch, $4 \mathrm{~mm}$; static touch, $5 \mathrm{~mm}$ ) spanned about five receptor spacings in each sensory system, indicating that the dimensional equivalence of vision and touch is determined by re ceptor spacing rather than central factors. The patterns of letter confusions and the productmoment correlations for correct identifications (vision vs. static touch, 0.83 ; vision vs. scanned touch, 0.75 ; and static touch v8. scanned touch, 0.89 , all taken at comparable performance levels) imply that similar spatial processes are involved in the different modes.
\end{abstract}

The tactual and visual letter-recognition experiments reported here were motivated by previous studies of the neural mechanisms underlying tactual spatial resolution (Johnson \& Lamb, 1981; Johnson \& Phillips, 1981; Phillips \& Johnson, 1981) that support the following working hypotheses: (1) Spatial resolution on the skin of the finger is limited by peripheral, rather than central, mechanisms. (2) This limit is determined by the innervation density and the spatiotemporal response properties of the SA (slowly adapting) mechanoreceptive fibers innervating the finger pad. (3) The increased richness of tactual sensation when the skin is scanned over a surface, as compared with stationary touch, is accounted for by two factors: (a) activation of two rapidly adapting mechanoreceptor populations that are silent when the skin is stationary, and (b) activation of the SA afferents at higher rates than when the skin is stationary. This activation of three mechanoreceptive afferent populations at high impulse rates provides an enriched basis for neural coding which parallels the subjective richness of tactual sensation during scanning.

It is important to distinguish tactual spatial resolution (e.g., letter recognition, Braille character recog-

J. R. Phillips's mailing address is: Department of Neuroscience, Johns Hopkins University School of Medicine, Baltimore, Maryland 21205. nition, grating orientation discrimination) as a subcategory within tactual spatial discrimination defined more generally. Many surfaces that are easily discriminated (e.g., grades of medium to fine sandpaper) differ in structural dimensions that are too fine to be resolved (e.g., as dots are resolved in a Braille pattern). The available experimental evidence (Lamb, 1983a, 1983b; Johnson, 1983) indicates that tactual spatial resolution depends on spatial neural imagery conveyed by the slowly adapting afferents, whereas fine texture discrimination depends on rate information conveyed by the rapidly adapting afferents.

These studies of the neural coding mechanisms underlying spatial tactual discrimination lead to specific predictions concerning discrimination behavior. For example, any form of discrimination behavior (e.g., texture discrimination) that is believed to depend on information relayed by the rapidly adapting afferents should be profoundly affected by skin movement. Katz was, we believe, referring to discrimination of this type when he observed that "movement is as indispensable to touch as light is to vision" (quoted in Krueger, 1970).

A second, unexpected prediction resulting from these neurophysiological studies is that spatial resolution should be similar whether the skin is held stationary or scanned across a surface. As stated above, the SA afferents appear to determine the resolution 
limits and they are active whether the skin is stationary or scanning. Furthermore, the spatial detail in the SA image evoked by a patterned stimulus (e.g., Braille) appears to be similar in either case (Johnson \& Lamb, 1981). Thus, according to the hypotheses set out above, spatial resolution should be similar whether the skin is stationary or moving. It was this prediction that motivated comparison of the two modes of touch in the experiments reported below.

The comparison of visual with tactual spatial resolution reported here was motivated by the marked similarities between tactual and visual letter recognition demonstrated by Craig (1979) and Loomis $(1981,1982)$. Craig has pointed out that one of the values of comparing spatial recognition performance in the two sensory systems is that extensive similarities probably imply that similar neural mechanisms are involved in processing spatial information in the two systems. The issue to be focused on here is the relationship between acuity and receptor spacing in the two systems.

Both vision and touch have preferred sites for spatial acuity: the fovea and finger pad, respectively. The available evidence suggests that in these regions the spatial acuity lies very close to the theoretical limits imposed by the Shannon sampling theorem in two dimensions (Rosenfeld \& Kak, 1976) and the receptor spacing. In the fovea of man, the cone spacing is about $0.45 \mathrm{~min}$ of arc (O'Brien, 1951). The effective receptor spacing in finger-pad skin is less easy to define, since several classes of mechanoreceptive afferents with different response properties innervate primate glabrous skin (Johansson \& Vallbo, 1979). However, on the basis of their spatial response properties, the slowly adapting (SA) afferents would have the greatest capacity to transmit the spatial detail of small, 3- to 8-mm, letters (Johnson \& Lamb, 1981; Phillips \& Johnson, 1981). The innervation density for SA afferents on the finger pad is approximately one afferent per millimeter (Johansson \& Vallbo, 1979). The two-dimensional sampling theorem implies that if the central mechanisms preserved the spatial information encoded by the first-order afferents perfectly, then the threshold periods for unambiguous spatial frequency resolution would be approximately $0.9 \mathrm{~min}$ of arc for vision and $2.0 \mathrm{~mm}$ for touch. Those figures are very close to the observed psychophysical results for grating resolution tasks (Campbell \& Green, 1965; Johnson \& Phillips, 1981), and they imply that both the visual and the somesthetic systems process spatial information from their regions of maximal spatial acuity with equal efficiency (i.e., with virtually no loss of spatial information). The experiments described here provide a basis for a direct comparison of visual and tactual performance using complex spatial stimuli near the limits of human spatial resolution in each system.

\section{METHOD}

\section{Subjects}

Four right-handed female students with at least normal Snellen visual acuity served as paid subjects in both the visual and tactual experiments. Experiments were conducted in sessions lasting $2 \mathrm{~h}$, each subject attending approximately 15 sessions.

\section{Visual Stimuli and Procedures}

Four sets of 26 black, sans serif capital letters (Helvetica) of different heights $(2.14,3.18,3.97$, and $4.76 \mathrm{~mm})$ but of approximately constant stroke width $(0.5 \mathrm{~mm})$ were used as visual stimuli. The corresponding letters of each set had equal height:width ratios. Each letter was positioned in the center of a white $10 \times 15 \mathrm{~cm}$ card and was presented for identification against a neutral background at a distance of $5.3 \mathrm{~m}$ from the subject, where the four sets of letters subtended visual angles of 1.5, 2.0, 2.5, and $3.0 \mathrm{~min}$, respectively. The cards were illuminated to give a reflected luminance of $6.0 \mathrm{~cd} / \mathrm{m}^{2}$. The subjects were told that each stimulus would consist of one capital letter that they were to identify and that all 26 letters were equally likely to be presented. The letters were presented in a pseudorandom order, such that in each session all 26 letters in a set had an equal probability of occurrence. The letters remained visible until the subject responded, after which the next stimulus was presented. No equivocal responses were allowed and at no time was training or feedback given. Each subject received 20 presentations of each letter at each size.

\section{Tactual Stimull and Procedures}

The tactual stimuli consisted of four sets of 26 embossed, sans serif capital letters, similar in form to the visual stimuli. Each set of letters was of a different height $(3.0,4.5,5.5$, and $8.0 \mathrm{~mm})$ but of approximately constant stroke width $(0.8 \mathrm{~mm})$. The letters were machined from plastic so that the letter faces were raised $1.5 \mathrm{~mm}$ above the background in order that the skin would not contact the background when the letters were presented.

The subject sat with her right arm resting on a table, with the palm of the hand facing downwards. Her right index finger extended through a slot in a screen that shielded all apparatus from her view. The letters were fixed near the edge of a large horizontal disk that could be rotated by the experimenter between trials to bring any letter beneath the subject's fingerpad. When in position, the letter lay 6 to $7 \mathrm{~mm}$ away from the skin, its vertical axis parallel with the axis of the finger (the top of the letter distally). Tactual stimuli were presented under two conditions, static and scanned. In the static condition, the disk was counterbalanced so that each letter, when in position below the finger, required a force of $60 \mathrm{~g}$ to depress it from a stop. The finger was constrained to allow only vertical movements, and the subject was required to identify each letter by actively lowering the finger, depressing the letter, and holding a constant displacement against the reaction force of $60 \mathrm{~g}$. This force was selected because the range of forces preferred by the subjects when identifying the largest letters was found to be 30 to $80 \mathrm{~g}$. When presented in the scanned condition, the disk was locked, the finger was not constrained, and the subject was free to tactually explore (scan) the letter with the finger pad with any force, velocity, and direction. The subject was told that each stimulus would consist of one embossed capital letter and that all letters were equally likely to be presented. At 15 -sec intervals, a light signaled a 4-sec period, during which the subject was required to depress the letter (or scan it, in the scan mode) and to identify it. The repetition rate and stimulus period were selected to give the experimenter time to record the response and position the next stimulus beneath the subject's finger.

The subjects rarely required the full 4-sec stimulus period to identify letters; they generally removed the finger and responded after only 2 to $3 \mathrm{sec}$. No equivocal responses were allowed, and no training or feedback was given. Each subject received 20 presentations of each letter at each letter size in both the static and scan conditions. 


\section{RESULTS}

\section{Spatial Equivalence Between Touch and Vision}

No substantial improvement in recognition performance was evident during the period of data collection, so the subjects' responses were summarized in 48 confusion matrices [one for each subject (4), in each mode (3), and at each letter size (4)]. Initially, subject performance under each condition was assessed as the mean probability of correct identification over all letters, computed from the diagonal cells for each matrix.

Figure 1 illustrates the performance of each of the four subjects, expressed as percent correct identification versus letter size, when the letters were presented in the visual and two tactual modes. In Figure 1, visual and tactual letter height have been scaled according to the receptor spacings in the two systems; that is, the abscissas for visual and tactual letter height have been adjusted so that $0.45 \mathrm{~min}$ of arc (the cone spacing in the fovea) is equivalent to $1.0 \mathrm{~mm}$ on the skin of the finger pad (the approximate spacing of SA primary mechanoreceptive afferents on the finger pad). The resultant overlap of the visual and tactual performance curves illustrates that the equivalent dimensions for spatial resolution in the two systems are approximately those of their receptor spacings. (This equivalence is discussed further in relation to Figure 2). Figure 1 also illustrates that for every subject and at all letter sizes tested, tactual letter recognition is consistently better for scanned than for static touch $\left(p=2^{-15}\right.$, sign test). The curves relating to static touch are similar to those obtained by Loomis (1981) for tactual recognition of letters over a similar

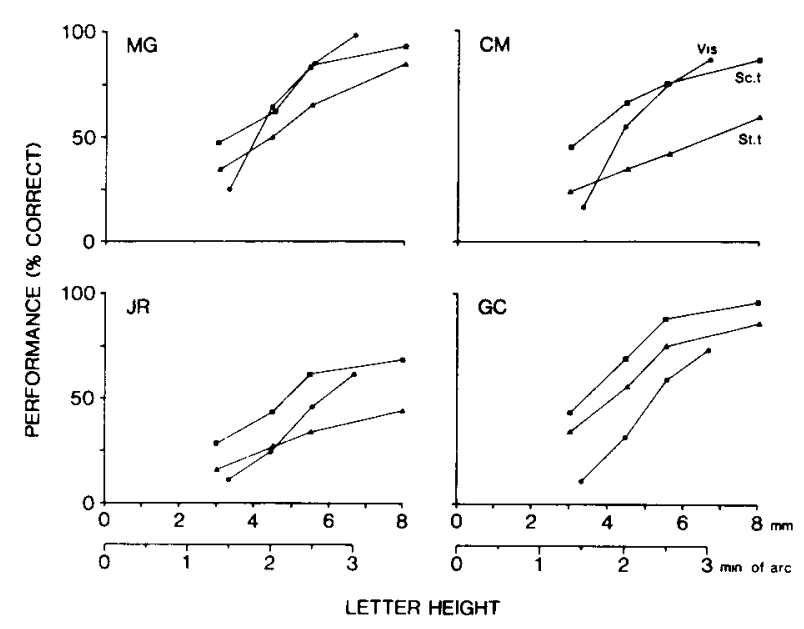

Figure 1. The performance (\% correct) of the four subjects versus letter height, for recognition of letters visunlly (filled circles), by static touch (triangles), and by scanned touch (squares). In each case, visual and tactual letter height have been scaled so that $0.45 \mathrm{~min}$ of arc is equivalent to $1.0 \mathrm{~mm}$ (1.e., the ratio of receptor spacings in the two systems). size range. At each letter size, the mean performance of his subjects was only about $10 \%$ lower than the mean performance of subjects in this study, even though letters were presented at a much lower force (about $10 \mathrm{~g}$ ) than that used in this study $(60 \mathrm{~g}$ ).

In order to obtain the spatial equivalence between vision and touch directly, the mean performance of subjects at each letter size and in each mode was computed, and the data were plotted on common axes. The letter sizes corresponding to $50 \%$ correct responses for each mode were normalized, and the three abscissas of Figure 2 were scaled accordingly. Thus, $2.2 \mathrm{~min}$ for vision, $5.2 \mathrm{~mm}$ for static touch, and $3.7 \mathrm{~mm}$ for scanned touch have been normalized, and the three abscissas in Figure 2 show the spatial equivalence between vision and the two tactual modes. On this basis, $1.0 \mathrm{~min}$ at the eye is the spatial equivalent of $2.4 \mathrm{~mm}$ on the distal finger pad for static touch. The spatial equivalence between the two tactual conditions is also apparent from Figure $2 ; 1.0 \mathrm{~mm}$ for static touch and $0.75 \mathrm{~mm}$ for scanned touch are approximately equivalent dimensions.

Figure 2 also illustrates that fractional increases in letter size do not have the same effect on performance in vision and touch. At a performance level of $50 \%$ correct, the slope of the vision curve is approximately twice as steep as either of those relating to touch. This difference in slope implies that the dimensional equivalence for vision and touch obtained in this way depends upon the performance level at which the data are normalized. For example, when normalized at performance levels below $50 \%$, the skin is relatively more acute; the dimensional equivalent to $1.0 \mathrm{~min}$ of arc is reduced to approximately $1.75 \mathrm{~mm}$ at $25 \%$ correct. However, at performance levels above $50 \%$, the skin is relatively less acute, and the equivalent dimension is increased to approximately $3.0 \mathrm{~mm}$ at $75 \%$ correct.

\section{Comparison of Visual and Tactual Confusion Matrices}

Construction of whole alphabet confusion matrices for vision and touch allows comparison of the two modalities across a large number of spatially different stimuli. Patterns of confusion between letters as well as the relative ease of identification of each letter may be compared as an indication of whether geometric form is processed in a similar manner in the two systems. It is assumed that the relative scores in the diagonal cells of a confusion matrix represent the relative legibility of letters not significantly contaminated by response bias (Loomis, 1982). Figure 3 illustrates confusion matrices obtained for vision (2.0-min letters) and static touch (4.5-mm letters). They represent the pooled data of four subjects, and both relate to a similar level of performance $(43 \%$ correct for vision, $42 \%$ correct for touch). Comparison of the diagonal cells of each matrix indicates 


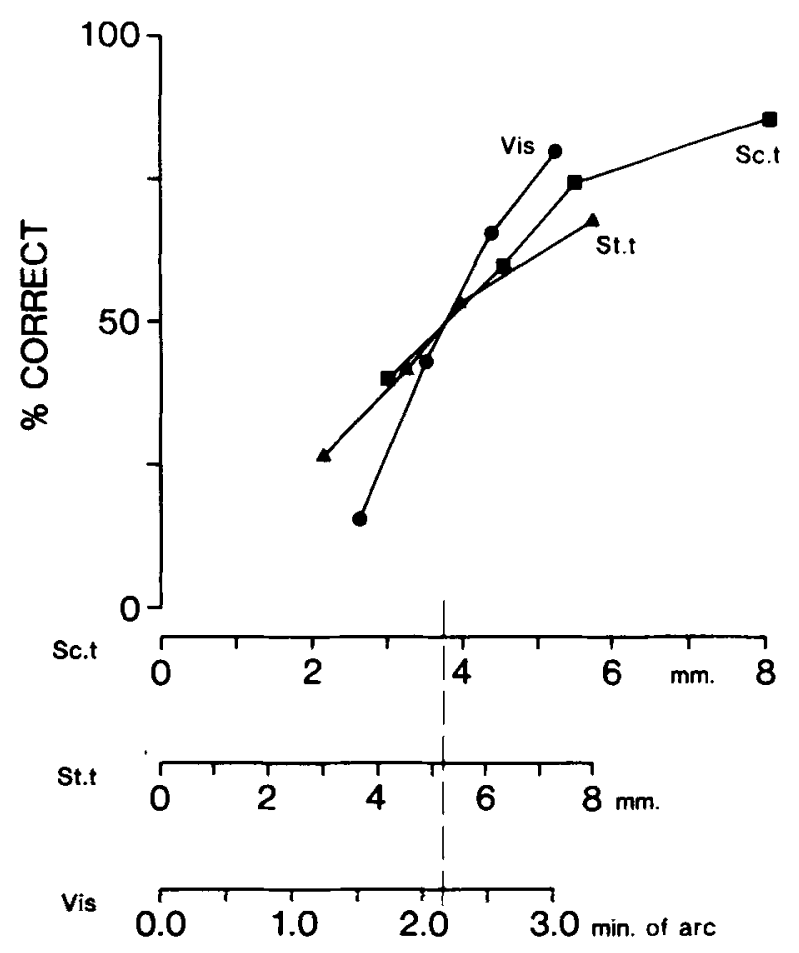

LETTER HEIGHT

Figure 2. The mean performance for vision (Vis), static touch (St.t), and scanned touch (Sc.t). Letter heights for $\mathbf{5 0 \%}$ correct responses in each mode have been normalized (vertical dashed line). The resultant alignment of the abacissa show the spatial equivaleace of the three modes.

that, at this level of performance, those letters that were most easily recognized visually were generally the most easily recognized tactually. Visually, the most easily recognized letters were $L, I, J, T, A, C$, and $W$. Tactually, the most easily recognized were I, J, L, A, T, W, and P. Similarly, the most difficult were $B, S, R, G, X, Y$, and $H$ for vision and $G, B$, $Q, M, N, R$, and $S$ for static touch.

The similarity between matrix diagonals (i.e., patterns of correct identifications) was assessed by the product-moment correlation coefficient:

$$
P_{k l}=\frac{\sigma_{k l}}{o_{k} \sigma_{1}}
$$

in which $\sigma_{\mathrm{kl}}$ represents the covariance and $\sigma_{\mathrm{k}}$ and $\sigma_{1}$ represent the standard deviations of the probabilities of correct identifications for matrices $k$ and 1 , respectively (see Appendix).

Initially, all possible correlations between individual subjects' data, at all letter sizes and for each sensory mode, were determined, yielding 1,128 correlation coefficients. Values of these coefficients spanned a wide range $(+0.95$ to -0.05$)$, the lower correlation values resulting from matrices representing widely disparate performance levels (range: $11 \%$ to $98 \%$ correct). When data for similar performance levels were considered alone, each subject showed the highest correlations between data from adjacent letter sizes within the same modality, then between data for static and scanned touch, then between static touch and vision, and the lowest between scanned touch and vision. This consistent ranking by all subjects is statistically significant (Kendall's coefficient of concordance $W=1.0, p=.005$, Siegel, 1956). These trends are apparent in Table 1 for the correlation coefficients between matrix diagonals obtained when the data of the four subjects were pooled. The correlations shown in Table 1 (and Table 2) are all significantly different from zero at the .01 level (Beyer, 1976). The highest correlation between vision and touch for pooled data $(+0.83)$ was obtained between 2.0-min letters for vision and 4.5-mm letters for static touch. The confusion matrices obtained for those letter sizes are shown in Figure 3.
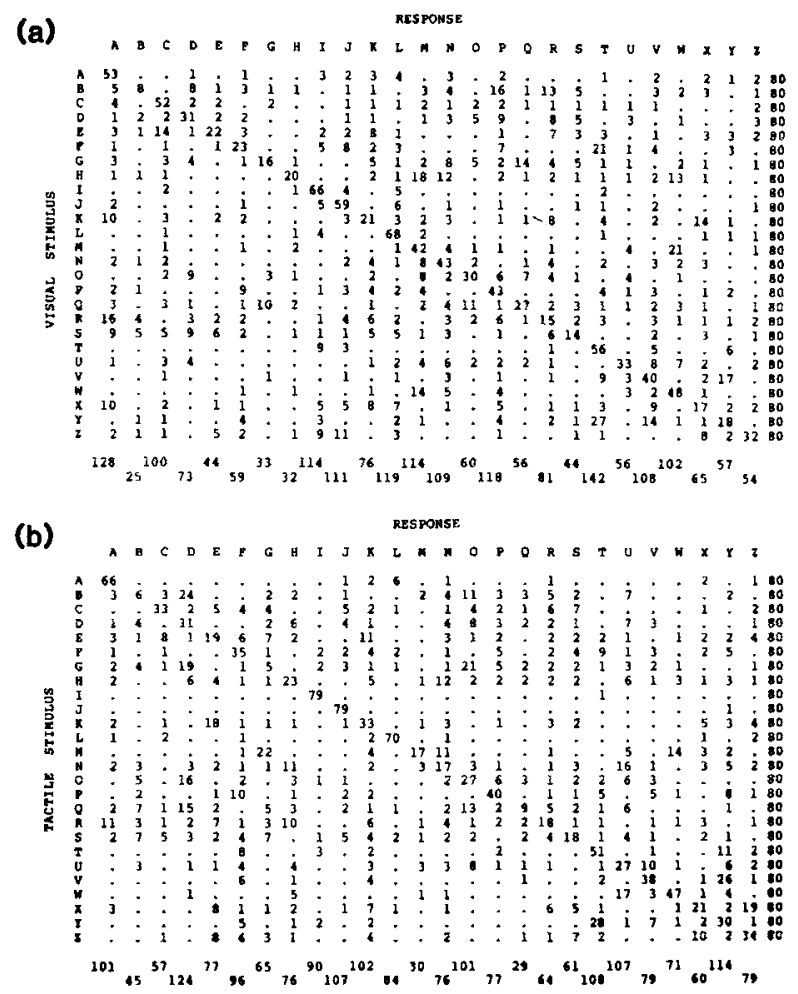

Figure 3. Confusion matrices constructed from the pooled data of the four subjects for (a) visual and (b) static tactual recognition of letters. Visual data for letter height $(2.0 \mathrm{~min})$ resulted in mean performance of $\mathbf{4 3} \%$ correct. Static tactual data for letter height $(4.5 \mathrm{~mm})$ resulted in $42 \%$ correct responses. In each matrix, the Itght-hand column shows the total number of times each letter was presented, and the bottom (stuggered) row lists the number of times ench response was evoked. 
Table 1

Correlation Coefficients for Confusion Matrix Diagonals Computed Between Modes and Between Adjacent Letter Heights Within Modes

\begin{tabular}{|c|c|c|c|c|c|c|c|}
\hline & & \multicolumn{2}{|c|}{ Vision (min arc) } & \multicolumn{2}{|c|}{ Static Touch (mm) } & \multicolumn{2}{|c|}{ Scanned Touch (mm) } \\
\hline & & 2.0 & 2.5 & 4.5 & 5.5 & 3.0 & 4.5 \\
\hline Vision (min arc) & $\begin{array}{l}2.0 \\
2.5\end{array}$ & $43 \%$ & $\begin{array}{l}0.85 \\
66 \%\end{array}$ & $\begin{array}{l}0.83 \\
0.68\end{array}$ & $\begin{array}{l}0.75 \\
0.61\end{array}$ & $\begin{array}{l}0.75 \\
0.58\end{array}$ & $\begin{array}{l}0.60 \\
0.52\end{array}$ \\
\hline Static Touch (mm) & $\begin{array}{l}4.5 \\
5.5\end{array}$ & & & $42 \%$ & $\begin{array}{l}0.95 \\
54 \%\end{array}$ & $\begin{array}{l}0.89 \\
0.85\end{array}$ & $\begin{array}{l}0.83 \\
0.88\end{array}$ \\
\hline Scanned Touch (mm) & $\begin{array}{l}3.0 \\
4.5\end{array}$ & & & & & $41 \%$ & $\begin{array}{l}0.87 \\
60 \%\end{array}$ \\
\hline
\end{tabular}

Note-Letter heights selected to span $50 \%$ correct identification. Diagonal entries show performance (percentage correct). Data are for pooled confusion matrices.

Table 2

Correlation Coefficients for Confusion Matrix Off-Diagonals Computed Between Modes and Between Adjacent Letter Heights Within Modes

\begin{tabular}{|c|c|c|c|c|c|c|c|}
\hline & & \multicolumn{2}{|c|}{ Vision (min arc) } & \multicolumn{2}{|c|}{ Static Touch (mm) } & \multicolumn{2}{|c|}{ Scanned Touch (mm) } \\
\hline & & 2.0 & 2.5 & 4.5 & 5.5 & 3.0 & 4.5 \\
\hline Vision (min arc) & $\begin{array}{l}2.0 \\
2.5\end{array}$ & $43 \%$ & $\begin{array}{l}0.67 \\
66 \%\end{array}$ & $\begin{array}{l}0.46 \\
0.43\end{array}$ & $\begin{array}{l}0.50 \\
0.42\end{array}$ & $\begin{array}{l}0.44 \\
0.38\end{array}$ & $\begin{array}{l}0.35 \\
0.34\end{array}$ \\
\hline Static Touch (mm) & $\begin{array}{l}4.5 \\
5.5\end{array}$ & & & $42 \%$ & $\begin{array}{l}0.73 \\
54 \%\end{array}$ & $\begin{array}{l}0.72 \\
0.76\end{array}$ & $\begin{array}{l}0.70 \\
0.85\end{array}$ \\
\hline Scanned Touch (mm) & $\begin{array}{l}3.0 \\
4.5\end{array}$ & & & & & $41 \%$ & $\begin{array}{l}0.78 \\
60 \%\end{array}$ \\
\hline
\end{tabular}

Note-Letter heights and performance values are as in Table 1.

Table 3

Numbers of Letters (of 26) Having the Same Primary Distractor When Data for Different Letter Sizes Were Compared Within and Across Modes

\begin{tabular}{|c|c|c|c|c|c|c|c|}
\hline & & \multicolumn{2}{|c|}{ Vision (min arc) } & \multicolumn{2}{|c|}{ Static Touch (mm) } & \multicolumn{2}{|c|}{ Scanned Touch (mm) } \\
\hline & & 2.0 & 2.5 & 4.5 & 5.5 & 3.0 & 4.5 \\
\hline Vision (min arc) & $\begin{array}{l}2.0 \\
2.5\end{array}$ & $43 \%$ & $\begin{array}{r}15 \\
66 \%\end{array}$ & $\begin{array}{l}8 \\
5\end{array}$ & $\begin{array}{l}6 \\
4\end{array}$ & $\begin{array}{l}7 \\
6\end{array}$ & $\begin{array}{l}6 \\
4\end{array}$ \\
\hline Static Touch (mm) & $\begin{array}{l}4.5 \\
5.5\end{array}$ & & & $42 \%$ & $\begin{array}{r}16 \\
54 \%\end{array}$ & $\begin{array}{l}13 \\
18\end{array}$ & $\begin{array}{l}15 \\
18\end{array}$ \\
\hline Scanned Touch (mm) & $\begin{array}{l}3.0 \\
4.5 \\
\end{array}$ & & & & & $41 \%$ & $\begin{array}{r}17 \\
60 \%\end{array}$ \\
\hline
\end{tabular}

Note-Letter heights and performance values are as in Table 1.

The matrices in Figure 3 also illustrate that, when erroneous judgments were made in either modality, letters were generally confused with a small number of alternatives. If the specific confusions (off-diagonal scores) made in vision and touch are similar, that would further support the idea that similar neural processes are involved in the two systems. Table 2 lists the correlation coefficients computed for the off-diagonal cells of the matrices constructed from pooled data. The values indicate that there are some similarities in tactual and visual letter confusions. The correlations in Table 2 were computed without symmetrizing the matrices and are appreciably lower than those reported by Loomis (1982) for symmetrized matrices. Symmetrizing the matrices from this study resulted in an increase (up to $45 \%$ ) in every coefficient corresponding to those in Table 2.

In order to further investigate the similarity of visual and tactual letter confusions, the primary distractor (the most common erroneous response) was determined for each letter at each size, for each mode, from the pooled data of the four subjects. These confusions were then compared across letter sizes within and between modalities. Table 3 shows the number of these common distractors for the combinations of letter size and modality displayed in Tables 1 and 2. For example, when the pooled confusion matrices for vision at $\mathbf{2 . 0}$ min and static touch 
at $4.5 \mathrm{~mm}$, illustrated in Figure 3, where compared, eight letters (A, F, O, P, R, U, V, Y) were found to have the same distractors. As can be seen in Table 3, the confusions within the touch modes were very similar between adjacent letter sizes and between modes. It is remarkable that between scanned touch at $4.5 \mathrm{~mm}$ and static touch at $5.5 \mathrm{~mm}$ (similar performance levels) only eight letters had different primary distractors. Also, as can be seen in Table 3, the number of common distractors between vision and touch is less than that between the touch modes or between the vision results at 2.0 and $2.5 \mathrm{~min}$. One aspect of the data, not illustrated in the table, is that, when common distractors are compared at disparate sizes within modes, the number of common distractors is greatly reduced (e.g., 3 between vision matrices at 1.5 and $3.0 \mathrm{~min} ; 6$ between static touch matrices at 3.0 and $8.0 \mathrm{~mm}$; and 10 between scanned touch matrices at 3.0 and $8.0 \mathrm{~mm}$ ).

\section{DISCUSSION}

The objectives of this study were to compare letterrecognition performance for two modes of touch and between touch and vision. The aim in the tactualtactual comparison was to quantify the effect of scanning on spatial resolution and to compare form perception in the two tactual modes (scanning vs. stationary touch). The aim in the tactual-visual comparison was to establish comparable spatial dimensions and to assess the similarity of form perception in the two systems.

\section{Scanning versus Static Touch}

Experiments comparing spatial resolution during scanning and static touch were undertaken as a test of a set of hypotheses concerning the neural mechanisms underlying tactual spatial discrimination (see the introduction and Johnson, 1983). Those hypotheses led to the prediction that tactual spatial resolution should be similar whether the skin is scanned or stationary. Briefly, the argument was that (1) tactual spatial resolution is limited by peripheral rather than central neural factors, (2) the slowly adapting (SA) afferents convey the spatial imagery used by the central nervous system in spatial resolution, (3) the SA afferents are active whether the skin deformation is stationary or moving, and (4) the spatial resolution of the afferent neural image carried by the SA population is unaffected by movement; therefore, tactual spatial resolution should be similar whether the skin is stationary or scanned. The results reported here bear out that prediction: the subjects' performance during scanning and static touch was similar (Figure 2 and Tables 1, 2, and 3). Letter resolution was approximately $25 \%$ more acute during scanning (comparable levels of performance were achieved for dimensions approximately $25 \%$ smaller; see Figure 2).

The small, but significant, improvement in subjects' performance cannot be explained with certainty, but there are two likely explanations. First, the SA afferents are much more sensitive to dynamic than to stationary deformation of the skin. This dynamic sensitivity results in much higher impulse rates during scanning, yielding a "brighter" neural image. The specific experimental observations were that scanned Braille dots evoked impulse rates, in SA afferents innervating the skin of a monkey finger pad, that were approximately 10 times greater than those evoked by stationary Braille dots indenting the skin; that is, the rates over the neural image ranged from 0 to 300 impulses per second (ips) rather than

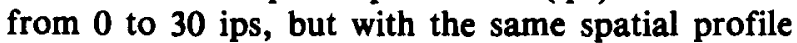
(Johnson \& Lamb, 1981). The result is a brighter image, which may explain the improved performance. The second explanation, which is not mutually exclusive, relates to the spatial information provided by a set of fixed sensors scanned across a fixed image. Given a sufficient temporal acuity for the sensors and an appropriate central integration mechanism, it is possible to reconstruct a spatial image that is much more detailed than that provided by the stationary sensory array. The intended mechanism can be visualized by holding a perforated sheet in front of an object, first motionless and then scanned horizontally. If the scanning is fast enough and the perforations are sufficiently dense, the spatial detail, which is only partially sensed at any one instant, becomes quite clear. Experiments by Craig (1982), Loomis (1980), and Taenzer (1970), using the Optacon, demonstrate the existence of spatiotemporal integration mechanisms within the somatosensory system. Whether they account for some or all of the improvement observed in the experiments reported here is problematical. Issues concerning the neural mechanisms of tactual discrimination during stationary and scanned touch are discussed in greater detail in Johnson (1983) and Johnson and Lamb (1981).

\section{Touch versus Vision}

Equivalent dimensions for touch and vision. The level of performance ( $50 \%$ correct) obtained for visual recognition of letters subtending $2.2 \mathrm{~min}$ was the same as that obtained for static tactual recognition of 5.2-mm letters. Thus, at that level of performance, $0.45 \mathrm{~min}$ at the eye is the spatial equivalent of $1.06 \mathrm{~mm}$ on the finger pad for static touch. That equivalence can be well accounted for in terms of the relative center-to-center spacing of receptors on the retina and fingertip; that is, $0.45 \mathrm{~min}$ of arc at the fovea and approximately $1.0 \mathrm{~mm}$ for the fingertip (see the introduction). In addition, the data of Figures 1 and 2 imply that the limits of spatial resolution 
for both touch and vision are determined by peripheral receptor spacing and not by any limitations in central neural mechanisms. The letter heights yielding $50 \%$ correct response span about five receptor spacings in each system. This is the expected result, because punctate arrays (e.g., dot matrix displays) fail to represent most letters unambiguously when letter heights are reduced to span less than five punctate spacings. Clearly, if performance is as good as the limitations of peripheral mechanisms allow, then there is little room for central processes that degrade spatial information.

Simple proportional scaling between touch and vision does not account for all the facts, however. When the psychometric functions for recognition accuracy in the two systems are scaled, as in Figure 2, the function for vision is steeper than for either touch mode, indicating that factors other than sensor spacing are significant determinants of spatial resolution in one or both systems. The same trend is evident in the data presented by Loomis (Figure 5 in Loomis, $1981)$, even though the visual stimuli were large (10 to $30 \mathrm{~min}$ ), blurred letters.

Similarities of form perception in the two systems. The data presented here (Tables 1-3) are comparable with those reported by Craig (1979) and Loomis (1982), and they imply that the processes underlying geometric form perception in the tactual and visual systems are similar but not identical. The similarity is evident from the high correlations between recognition performance and distractors in the two systems. That the processes are not identical is indicated by the fact that the correspondences are not as high between visual and tactual performance as between the two touch modes. However, form perception in the two systems is clearly more similar than different. Thus (as Craig, 1979, has argued), theories of form discrimination should focus on common mechanisms rather than on mechanisms found in only one of the systems (e.g., simple, complex, and hypercomplex neuronal response properties).

\section{REFERENCES}

BEYEn, W. H. Handbook of tables for probability and statistics. Cleveland: The Chemical Rubber Co., 1976.

Campbell, F. W., \& Green, D. G. Optical and retinal factors affecting visual resolution. Journal of Physiology (London), 1965, 181, 576-593.

Crarg, J. C. A confusion matrix for tactually presented letters. Perception \& Psychophysics, 1979, 26, 409-411.

Crajo, J. C. Temporal integration of vibrotactile patterns. Perception \& Psychophysics, 1982, 32, 219-229.

Johansson, R. S., \& Valleo, A. B. Tactile sensibility of the human hand: Relative and absolute densities of four types of mechanoreceptive units in glabrous skin. Journal of Physiology (London), 1979, 286, 283-300.

JoHnson, K. O. Neural mechanisms of tactual form and texture discrimination. Federation Proceedings, 1983, 42, 2542-2547.

Johnson, K. O., \& LAMB, G. L. Neural mechanisms of spatial tactile discrimination: Neural patterns evoked by Braille-like dot patterns in the monkey. Journal of Physiology (London), $1981,310,117-144$.

Johnson, K. O., \& Phillips, J. R. Tactile spatial resolution: I. Two point discrimination, gap detection, grating resolution and letter recognition. Journal of Neurophysiology, 1981, 46, 1177-1191.

Krueger, L. E. David Katz's Der Aufbau der Tastwelt (The world of touch): A synopsis. Perception \& Psychophysics, 1970, 7, 337.341.

Lamb, G. Tactile discrimination of textured surfaces: Psychophysical performance measurements in humans. Journal of Physiology (London), 1983, 338, 551-565. (a)

LAMB, G. Tactile discrimination of textured surfaces: Peripheral neural coding in the monkey. Journal of Physiology (London), 1983, 338, 567-587. (b)

Loomıs, J. M. Interaction of display mode and character size in vibrotactile letter recognition. Bulletin of the Psychonomic Society, 1980, 16, 385-387.

Loomis, J. M. On the tangibility of letters and braille. Perception \& Psychophysics, 1981, 29, 37-46.

Lоoмis, J. M. Analysis of tactile and visual confusion matrices. Perception \& Psychophysics, 1982, 31, 41-52.

O'BRIEN, B. Vision and resolution in the central retina. Journal of the Optical Society of America, 1951, 41, 882-894.

Phillips, J. R., \& Johnson, K. O. Tactile spatial resolution: II. Neural representation of bars, edges and gratings in monkey primary afferents. Journal of Neurophysiology, 1981, 46, 1192-1203.

Rosenfeld, A., \& KAK, A. C. Digital picture processing. New York: Academic Press, 1976.

Siegel, S. Nonparametric statistics. New York: McGraw-Hill, 1956.

TAEnzer, J. C. Visual word reading. IEEE Transactions on ManMachine Systems, 1970, 11, 44-53.

\section{APPENDIX}

The variances and covariances in the formula for correlation between the diagonal elements of two confusion matrices (the probabilities of correct identification) are estimated as follows. Let $P_{i j k}$ represent the probability that letter $i$ is identified as letter $j$ in the $k^{\text {th }}$ confusion matrix. Then, the mean probability of correct identification in matrix $k$ is given by

$$
m_{k}=\frac{1}{n} \sum_{i=1}^{n} P_{i j k}
$$

where $\mathrm{n}=26$; the variance of identification probabilities is given by:

$$
\sigma_{k}^{2}=\frac{1}{(n-1)} \sum_{i=1}^{n}\left(p_{i j k}-m_{k}\right)^{2}
$$

and the covariance of identification probabilities between matrices $k$ and $l$ by:

$$
\sigma_{k l}=\frac{1}{(n-1)} \sum_{i=1}^{n}\left(p_{i j k}-m_{k}\right)\left(p_{i j l}-m_{1}\right)
$$

(Manuscript received January 20, 1983; revision accepted for publication May 24, 1983.) 